Bull. Fac. Agric., Cairo Univ., 58 (2007): 11-20.

\title{
ESTIMATION OF LOCATIONS AND GENOTYPES X LOCATIONS VARIANCES USING DIFFERENT APPROACHES OF ANALYSING RANDOMIZED COMPLETE BLOCK DESIGN
}

(Received:18.5.2006)

\author{
By \\ H. A. Idris , S. M. Seyam and S. S. Badr \\ Cotton Research Institute, Agriculture Research Center, Giza, Egypt.
}

\begin{abstract}
The present investigation is dealing with the variances of five long staple Egyptian cotton (Gossypium barbadense L.) genotypes, with respect to yield, its components and fiber properties in an old location (Middle Delta) and a new location (North Delta) during 2004 and 2005 seasons. The final goal is to study the possibility of suggestion a modified analysis of randomized complete block design to replace the use of combined analysis. The five genotypes were cultivars, viz. G.85, G.86 and G.89, the others were hybrids, viz (G.89 x G.86) and (G.89 x Pima S-6). Modified analysis depends on the use of the five genotypes twice, however the number of the replicated genotypes remains the same. The modified analysis gave equal results as the traditional combined. In addition the modified analysis does not need using the Bartlett test .
\end{abstract}

Key words: cotton,, (genotypes $x$ locations), locations, randomized complete block design.

\section{INTRODUCTION}

Agricultural experiments, such as variety tests, seed treatment experiments, cultural practice determinations, and related field research are usually repeated in various locations for a number of seasons. This is necessary because the effects of most variables or factors considerably vary from location to another as well as from season to season. In this type of agricultural field experimentation it is possible to determine the effects of environment and location on the treatments being studied. As a consequence of the repetitions of the experiments at locations and seasons, it is also possible to make more widely applicable recommendations. Thus, researchers need a developed statistical measure to estimate the locations and (genotypes $\mathrm{x}$ locations) variances.

Idris (2002) evaluated some Egyptian cotton cultivars in Middle Delta using combined randomized complete block design (CRCBD). He found that the mean squares for locations, cultivars and the interaction between them were significantly different with respect to yield and its components. El Oraby (2003) studied six Egyptian cotton genotypes in North Delta using (CRCBD). $\mathrm{He}$ reported that the mean squares for (genotypes $\mathrm{x}$ locations) significantly varied according to yield (seed and lint), boll weight, fiber length and micronaire reading. Mohamed (2005) evaluated ten Egyptian cotton genotypes in both Middle and North Delta by (CRCBD). The results indicated that the mean squares for locations and (genotypes $\mathrm{x}$ locations) were significantly different for aspects of yield (seed and lint), boll weight, lint percentage, fiber length and micronaire reading. Idris (2005) studied five Egyptian cotton genotypes in two locations by using two steps of analyses randomized complete block design. Such steps considered each location as one replicate. The results showed no difference between the two ways of analyses with respect to locations effects. $\mathrm{He}$ added that the interaction (genotypes $\mathrm{x}$ locations) was significant for combined analysis, the genotypes exhibited no significance for the two steps.

The objective of the present study was to estimate the locations and genotypes $\mathrm{x}$ locations variances using different approaches of analysing randomized complete block design.

\section{MATERIALS AND METHODS}

Eight field experiments were carried out in two different locations, (old location and new location) during 2004 and 2005 seasons. In each season, two experiments were conducted in each location. For the old one (Middle Delta), Sharkia and Gharbia Governorates, meanwhile in the new locations (North Delta), Domiatta and Kafr El Sheikh Governorates. A randomized complete 
block design with 4 replications was used in each experiment. Planting was during the last week of March. All other cultural practices were done as usual.

Five long staple Egyptian cotton (Gossypium barbadense L.) genotypes were grown. Three of them were cultivars (C), viz. G.85, G.86 and G.89. The two remainders were hybrids (H), viz. (G.89 $x$ G.86) and (G.89 x Pima S-6). In each experiment, the genotypes were evaluated with 4 replications for yield, its components and one replicate for fiber properties.

Yield were seed cotton yield (S.C.Y.) kentar/ faddan (k/fed.) and lint cotton yield (L.C.Y.) kentar/ feddan $(\mathrm{k} / \mathrm{fed})$. Yield components were boll weight (B.W.) gm, lint percentage (L.P.) \%, seed index (S.I.) gm. Fiber properties aspects were fiber length (F.L) mm, strength (St.) g/tex, and micronaire reading (Mic.). Fiber tests were done using (HVI).

\subsection{Statistical analysis of individual location}

The idea is to analyse each location using all replications ( $\mathrm{r}$ ) for the two experiments. Where $\mathrm{r}=$ $\Sigma(r)$ in the two Governorates in each location (old and new). Statistical analysis is straightforward as Cochran and Cox (1950), Federer (1955) and Gomez and Gomez (1984).

\subsection{Statistical analysis of modified randomized complete block design}

A modified analysis suggested by the author was used. In such proposal the data of the 5 genotypes were used twice. One from the old location $\left(\mathrm{G}_{1}\right)$, while the other from the new location $\left(G_{2}\right)$ giving 10 genotypes $(G)$ in two equal groups and $\left(\mathrm{G}_{1}\right)$ vs $\left(\mathrm{G}_{2}\right)$ to estimate locations and genotypes $\mathrm{x}$ locations variances. In such modified ANOVA the number of replicates remains the same as in each location, (Table 1). Homogeneity test of variances (Bartlett test) was not used before the analysis.

\subsection{Statistical analysis of combined randomized complete block design}

Traditional combined analysis depends on 5 genotypes and increased replicates to estimate locations and genotypes $\mathrm{x}$ location variances (Table 1). Homogeneity test of variances (Bartlett test) was used according to the procedures reported by Bailey (1994). All statistical procedures and methods were carried according to Roger (1994). The treatment means were compared by L.S.D. test as given by Steel and Torrie (1980). All comparisons were done at 0.05 level of significance.

Table (1): Comparison between modified and combined randomized complete block design analyses.

\begin{tabular}{|c|c|c|c|}
\hline \multicolumn{2}{|c|}{ Modified analysis } & \multicolumn{2}{c|}{ Combined analysis } \\
\hline Source of variation & d.f. & Source of variation & d.f. \\
\hline Replications $(\mathbf{r})$ & $(\mathrm{r}-1)$ & Locations $(\mathbf{L})$ & $(\mathrm{L}-1)$ \\
Genotypes $(\mathbf{G})$ & $(\mathrm{g}-1)$ & Rep. / Locations & $\mathrm{L}(\mathrm{r}-1)$ \\
Within old location $\left(\mathbf{G}_{\mathbf{1}}\right)$ & $\left(\mathrm{g}_{1}-1\right)$ & Genotypes $(\mathbf{G})$ & $(\mathrm{g}-1)$ \\
Within new location $\left(\mathbf{G}_{2}\right)$ & $\left(\mathrm{g}_{2}-1\right)$ & $\mathbf{G} \mathbf{x} \mathbf{L}$ & $(\mathrm{g}-1)(\mathrm{L}-1)$ \\
Old vs new & 1 & & \\
Experimental error & $(\mathrm{g}-1)(\mathrm{r}-1)$ & Experimental error & $\mathrm{L}(\mathrm{r}-1)(\mathrm{g}-1)$ \\
\hline Total & $\mathrm{g} \mathrm{r}-1$ & Total & $\mathrm{L} \mathrm{g} \mathrm{r}-1$ \\
\hline
\end{tabular}

\section{RESULTS AND DISCUSSION}

\subsection{Yield and its components}

\subsubsection{Analysis of individual location (one season)}

Each location considered 8 replications and analysis was a normal randomized complete block design. The analysis of variance revealed significant variation due to replications, genotypes and partitioning of genotypes, (Table 2).

In the first season, significant variation due to replications was observed for yield components in both locations except (B.W.) with respect to old location. Significant variation due to genotypes was detected on all traits except (L.C.Y.) in the old location. In contrast, in the new location, no significant variation due to genotypes was observed except for two traits, viz. (B.W.) and (S.I.). Significant differences due to cultivars were detected on (L.P.) in the old location, (B.W.) in the new location and (S.I.) in the two locations. Significant variation due to hybrids was observed for all traits in the old location except one trait, (L.P.). In both locations no significant variation due to cultivars vs. hybrid was detected with respect to yield and its components except (B.W.) in the new location.

In the second season, significant variation due to replications was detected on (B.W.) in the old location, yields (S.CY. and L.C.Y.) in the new location and (S.I.) in both locations. Significant difference due to genotypes was observed for yield components in the old location, (S.CY.), 
(L.C.Y.) and (L.P.) in the new location. Significant variation due to cultivars was detected on all traits except (S.C.Y.) and (S.I.) in the old location. In contrast, cultivars exhibited nonsignificant differences in the new location except (L.C.Y.) and (L.P.). Significant differences

Table (2): Mean squares of yield and its components for individual location (one season),

\begin{tabular}{|c|c|c|c|c|c|c|}
\hline \multicolumn{7}{|c|}{ Season 2004} \\
\hline \multicolumn{7}{|c|}{ Old location (Middle Delta) } \\
\hline \multicolumn{2}{|l|}{ Traits } & \multirow{2}{*}{$\begin{array}{l}\text { S.C.Y. } \\
\text { (k/fed) }\end{array}$} & \multirow{2}{*}{$\begin{array}{l}\text { L.C.Y. } \\
\text { (k/fed) }\end{array}$} & \multirow{2}{*}{$\begin{array}{l}\text { B.W. } \\
\text { (gm) }\end{array}$} & \multirow{2}{*}{$\begin{array}{l}\text { L.P. } \\
(\%)\end{array}$} & \multirow{2}{*}{$\begin{array}{l}\text { S. I. } \\
\text { (gm) }\end{array}$} \\
\hline Source of variation & d.f. & & & & & \\
\hline Replications & 7 & 2.13 & 4.33 & 0.043 & $3.65 * *$ & $0.448 *$ \\
\hline Genotypes & 4 & $8.15 *$ & 5.23 & $0.125 * *$ & $6.95 * *$ & $1.39 * *$ \\
\hline Cultivars (C) & 2 & 3.76 & 2.73 & 0.072 & $13.64 * *$ & $1.39 * *$ \\
\hline Hybrids (H) & 1 & $21.65 * *$ & $14.84 *$ & $0.334 * *$ & 0.461 & $2.27 * *$ \\
\hline C. vs H. & 1 & 3.43 & 0.618 & 0.023 & 0.072 & 0.493 \\
\hline Experimental error & 28 & 2.19 & 2.91 & 0.025 & 0.823 & 0.185 \\
\hline \multicolumn{7}{|c|}{ New location (North Delta) } \\
\hline \multicolumn{2}{|l|}{ Traits } & \multirow{2}{*}{$\begin{array}{l}\text { S.C.Y. } \\
\text { (k/fed) }\end{array}$} & \multirow{2}{*}{$\begin{array}{l}\text { L.C.Y. } \\
\text { (k/fed) }\end{array}$} & \multirow{2}{*}{$\begin{array}{l}\text { B.W. } \\
\text { (gm) }\end{array}$} & \multirow{2}{*}{$\begin{array}{l}\text { L.P. } \\
(\%)\end{array}$} & \multirow{2}{*}{$\begin{array}{l}\text { S. I. } \\
\text { (gm) }\end{array}$} \\
\hline Source of variation & d.f. & & & & & \\
\hline Replications & 7 & 3.81 & 7.78 & $0.357 * *$ & $5.84 * *$ & $1.34 * *$ \\
\hline Genotypes & 4 & 2.90 & 4.50 & $0.141 * *$ & 0.212 & $1.27 *$ \\
\hline Cultivars (C) & 2 & 1.38 & 3.85 & $0.167 * *$ & 0.410 & $1.99 * *$ \\
\hline Hybrids (H) & 1 & 2.81 & 4.90 & 0.001 & 0.004 & 0.744 \\
\hline C. vs H. & 1 & 6.03 & 5.42 & $0.231 * *$ & 0.027 & 0.381 \\
\hline Experimental error & 28 & 2.24 & 3.36 & 0.021 & 0.948 & 0.315 \\
\hline \multicolumn{7}{|c|}{ Season 2005} \\
\hline \multicolumn{7}{|c|}{ Old location (Middle Delta) } \\
\hline \multicolumn{2}{|l|}{ Traits } & \multirow{2}{*}{$\begin{array}{l}\text { S.C.Y. } \\
\text { (k/fed.) }\end{array}$} & \multirow{2}{*}{$\begin{array}{l}\text { L.C.Y. } \\
\text { (k/fed) }\end{array}$} & \multirow{2}{*}{$\begin{array}{c}\text { B.W. } \\
(\mathrm{gm})\end{array}$} & \multirow{2}{*}{$\begin{array}{c}\text { L.P. } \\
(\%)\end{array}$} & \multirow{2}{*}{$\begin{array}{c}\text { S. I. } \\
\text { (gm) }\end{array}$} \\
\hline Source of variation & d.f. & & & & & \\
\hline Replications & 7 & 0.515 & 1.25 & $0.049 *$ & 1.09 & $0.946 * *$ \\
\hline Genotypes & 4 & 0.814 & 3.75 & $0.127 * *$ & $9.50 * *$ & $1.47 * *$ \\
\hline Cultivars (C) & 2 & 1.48 & $6.80 *$ & $0.074 *$ & $15.74 * *$ & 0.599 \\
\hline Hybrids (H) & 1 & 0.191 & 1.38 & $0.123 *$ & $4.51 *$ & $2.17 * *$ \\
\hline C. vs H. & 1 & 0.105 & 0.008 & $0.238 * *$ & 2.00 & $2.51 * *$ \\
\hline Experimental error & 28 & 1.04 & 1.33 & 0.017 & 0.812 & 0.252 \\
\hline \multicolumn{7}{|c|}{ New location (North Delta) } \\
\hline \multicolumn{2}{|l|}{ Traits } & \multirow{2}{*}{$\begin{array}{l}\text { S.C.Y. } \\
\text { (k/fed) }\end{array}$} & \multirow{2}{*}{$\begin{array}{l}\text { L.C.Y. } \\
\text { (k/fed) }\end{array}$} & B.W. & L.P. & S. I. \\
\hline Source of variation & d.f. & & & (gm) & $(\%)$ & $(\mathbf{g m})$ \\
\hline Replications & 7 & $10.82 * *$ & $18.43 * *$ & 0.034 & 1.95 & $1.28 * *$ \\
\hline Genotypes & 4 & $3.91 *$ & $7.59 * *$ & 0.021 & $10.58 * *$ & 0.590 \\
\hline Cultivars (C) & 2 & 0.603 & $4.51^{*}$ & 0.004 & $20.17 * *$ & 0.807 \\
\hline Hybrids (H) & 1 & 0.034 & 0.601 & 0.037 & 0.838 & 0.019 \\
\hline C. vs H. & 1 & $14.41 * *$ & $20.72 * *$ & 0.040 & 1.12 & 0.727 \\
\hline Experimental error & 28 & 1.02 & 1.09 & 0.017 & 0.916 & 0.277 \\
\hline
\end{tabular}

*, ** Significant at 0.05 and 0.01 levels, respectively.

due to hybrids were observed for yield components in the old location. Significant variation due to cltivars vs. hybrids was detected on (B.W.) and (S.I.) in the old location and yields (S.CY. and L.C.Y.) in the new location.

The results indicated significance due to replications according to variance within each location. Hybrids exhibited non-significant variation for all traits in the new location indicated that responses to environmental factors were similar. In contrast, hybrids exhibited significant differences in the old location indicated different responses to environmental factors. Cultivars vs. hybrids exhibited non-significant variation for yield in the old location during the two seasons.

\subsubsection{Modified analysis (one season)}

The analysis of variance showed significant difference due to partitioning of genotypes, (Table 3 ). In both seasons, significant variation due to genotypes within the old location was detected for all traits except (S.C.Y.) in 2005 season and (L.C.Y.) in the two seasons. Genotypes within new location exhibited significant variation for two yield components, viz. (B.W.) and (S.I.) in the first season, yields and (L.P.) in the second season. Significant difference due to the old vs. 
the new were observed for yields during the two seasons, (S.I.) in the first season and (L.P) in the second season indicated that yields (S.C.Y. and L.C.Y.) were more affected locations than yield components.

\subsubsection{Combined analysis (one season)}

The analysis of variance revealed significant variation due to locations and (genotypes $\mathrm{x}$ locations) (Table 3). Significant difference on locations was observed for yields during the two seasons, (S.I.) in the first season and (L.P.) in the second season indicated that yields (S.C.Y. and L.C.Y.) were more affected than yield components. Significant variation due to (genotypes $\mathrm{x}$ locations) was detected for yield components in the first season, (L.C.Y.) and (S.I.) in the second season.

Table (3): Mean Squares of yield and its components for two ways of analysis randomized complete block design (one season).

\begin{tabular}{|c|c|c|c|c|c|c|}
\hline \multicolumn{7}{|c|}{ Season 2004} \\
\hline \multicolumn{7}{|c|}{ Modified analysis } \\
\hline \multicolumn{2}{|l|}{ Traits } & \multirow{2}{*}{$\begin{array}{l}\text { S.C.Y. } \\
\text { (k/fed) }\end{array}$} & \multirow{2}{*}{$\begin{array}{l}\text { L.C.Y. } \\
\text { (k/fed) }\end{array}$} & \multirow{2}{*}{$\begin{array}{l}\text { B.W. } \\
\text { (gm) }\end{array}$} & \multirow{2}{*}{$\begin{array}{l}\text { L.P. } \\
(\%)\end{array}$} & \multirow{2}{*}{$\begin{array}{l}\text { S. I. } \\
\text { (gm) }\end{array}$} \\
\hline Source of variation & d.f. & & & & & \\
\hline Replications & 7 & 4.40 & 7.04 & $0.153^{* *}$ & 0.461 & 0.615 \\
\hline Genotypes & 9 & $16.27 * *$ & $15.51 * *$ & $0.120 *$ & $3.94^{*}$ & $2.19 * *$ \\
\hline Within old location & 4 & $8.15 * *$ & 5.23 & $0.125^{*}$ & $6.95 * *$ & $1.39 * *$ \\
\hline Within new location & 4 & 2.90 & 4.50 & $0.141^{*}$ & 0.212 & $1.27 *$ \\
\hline Old vs. New & 1 & $102.20 * *$ & $100.67 * *$ & 0.010 & 6.79 & $9.10 * *$ \\
\hline Experimental error & 63 & 2.14 & 3.35 & 0.048 & 1.79 & 0.353 \\
\hline \multicolumn{7}{|c|}{ Combined analysis } \\
\hline \multicolumn{2}{|l|}{ Traits } & S.C.Y. & L.C.Y. & B.W. & L. P. & S. I. \\
\hline Source of variation & d.f. & (k/fed) & $(\mathrm{k} / \mathrm{fed})$ & $(\mathrm{gm})$ & $(\%)$ & $(\mathbf{g m})$ \\
\hline Locations (L) & 1 & $102.20 * *$ & $100.67 * *$ & 0.010 & 6.79 & $9.10 * *$ \\
\hline Rep. / Locations & 14 & 2.97 & 6.05 & 0.200 & 4.75 & 0.893 \\
\hline Genotypes (G) & 4 & $9.31 * *$ & $8.97 *$ & $0.203 * *$ & $4.66 * *$ & $1.63 * *$ \\
\hline GXL & 4 & 1.74 & 0.761 & $0.064 *$ & $2.50 *$ & $1.03 * *$ \\
\hline Experimental error & 56 & 2.22 & 3.14 & 0.023 & 0.886 & 0.250 \\
\hline \multicolumn{7}{|c|}{ Season 2005} \\
\hline \multicolumn{7}{|c|}{ Modified analysis } \\
\hline \multicolumn{2}{|l|}{ Traits } & S.C.Y. & L.C.Y. & B.W. & L.P. & S. I. \\
\hline Source of variation & d.f. & (k/fed) & (k/fed) & $(\mathbf{g m})$ & $(\%)$ & (gm) \\
\hline Replications & 7 & $5.85 * *$ & $9.37 * *$ & $0.076^{* *}$ & 0.391 & 0.457 \\
\hline Genotypes & 9 & $6.14 * *$ & $13.65 * *$ & $0.070 * *$ & $12.28 * *$ & $1.10 *$ \\
\hline Within old location & 4 & 0.814 & 3.75 & $0.127 * *$ & $9.50 * *$ & $1.47 *$ \\
\hline Within new location & 4 & $3.91 *$ & $7.59 *$ & 0.021 & $10.58 * *$ & 0.590 \\
\hline Old vs. New & 1 & $36.37 * *$ & $77.54 * *$ & 0.034 & $30.18 * *$ & 1.66 \\
\hline Experimental error & 63 & 1.52 & 2.22 & 0.016 & 1.06 & 0.431 \\
\hline \multicolumn{7}{|c|}{ Combined analysis } \\
\hline \multicolumn{2}{|l|}{ Traits } & S.C.Y. & L.C.Y. & B.W. & L. P. & S. I. \\
\hline Source of variation & d.f. & (k/fed) & (k/fed) & $(\mathrm{gm})$ & $(\%)$ & (gm) \\
\hline Locations (L) & 1 & $36.37 *$ & $77.54^{*}$ & 0.034 & $30.18 * *$ & 1.66 \\
\hline Rep. /Locations & 14 & 5.67 & 9.84 & 0.042 & 1.52 & 1.11 \\
\hline Genotypes (G) & 4 & 2.58 & $7.48 * *$ & $0.124 * *$ & $18.67 * *$ & $0.989 *$ \\
\hline GX L & 4 & 2.15 & $3.85^{*}$ & 0.025 & 1.40 & $1.07 * *$ \\
\hline Experimental error & 56 & 1.03 & 1.21 & 0.017 & 0.865 & 0.264 \\
\hline
\end{tabular}

\subsubsection{Analysis of individual location (two seasons)}

Each location considered 16 replications and analysis of normal randomized complete block design. The analysis of variance showed significant variation due to replications, genotypes and partitioning of genotypes, (Table 5).

In both locations, significant difference due to replications was observed for all traits. Significant variations due to genotypes and cultivars were detected on yield components in the two locations.
Significant variation due to hybrids was observed for all traits in the old location. Significant difference due to cultivars vs. hybrids was detected on only one trait, (B.W.) in both locations. The results indicated significance due to replications according to different environments within each location. The cultivars exhibited significant variation with respect to yield components in both locations due to genetic differences. Hybrids exhibited significant differences with respect to yield and its 
components in the old location indicated that the behavior of them was different and more affected by environmental factors within the old location. In contrast, they exhibited non-significant difference for all traits indicating similar behavior within the new location. Cultivars vs. hybrid exhibited significant variation for only one trait (B.W.) in both locations indicating that (B.W.) was more affected by different environments within each location than other traits.

Table (4): Means of yield and its components (one season).

\begin{tabular}{|c|c|c|c|c|c|c|}
\hline \multicolumn{7}{|c|}{ Season 2004} \\
\hline Traits & \multicolumn{2}{|c|}{ S. C. Y. (k/fed) } & \multicolumn{2}{|c|}{ L. C. Y. (k/fed) } & \multicolumn{2}{|c|}{ B. W. (gm) } \\
\hline Genotypes & Old & New & Old & New & Old & New \\
\hline G.85 & 12.29 & 10.12 & 14.99 & 12.55 & 3.13 & 3.15 \\
\hline G.86 & 12.82 & 10.92 & 16.12 & 13.93 & 3.22 & 3.29 \\
\hline G.89 & 13.65 & 10.71 & 15.81 & 13.12 & 3.03 & 3.00 \\
\hline G.89 x G.86 & 12.36 & 10.96 & 14.93 & 13.40 & 3.22 & 2.99 \\
\hline G.89 x Pima S-6 & 14.68 & 11.80 & 16.86 & 14.50 & 2.93 & 3.00 \\
\hline L.S.D. (I) & 1.48 & ---- & 1.71 & ---- & 0.16 & 0.14 \\
\hline L.S.D. (M) & 1.46 & ---- & ---- & ---- & 0.22 & 0.22 \\
\hline L.S.D. (C) & & & & & \multicolumn{2}{|c|}{0.15} \\
\hline Traits & \multicolumn{2}{|c|}{ L.P. (\%) } & \multicolumn{2}{|c|}{ S. I. (gm) } & & \\
\hline G.85 & 38.60 & 39.23 & 11.70 & 10.98 & & \\
\hline G.86 & 39.91 & 39.29 & 11.65 & 11.40 & & \\
\hline G.89 & 37.30 & 38.87 & 10.95 & 10.41 & & \\
\hline G.89 x G.86 & 38.35 & 39.20 & 12.04 & 10.52 & & \\
\hline G.89 x Pima S-6 & 38.69 & 39.18 & 11.28 & 10.95 & & \\
\hline L.S.D. (I) & 0.91 & ---- & 0.43 & 0.56 & & \\
\hline L.S.D. (M) & 1.34 & ---- & 0.59 & 0.59 & & \\
\hline L.S.D. (C) & \multicolumn{2}{|c|}{0.95} & \multicolumn{2}{|c|}{0.51} & & \\
\hline \multicolumn{7}{|c|}{ Season 2005} \\
\hline Traits & \multicolumn{2}{|c|}{ S. C. Y. (k/fed) } & \multicolumn{2}{|c|}{ L. C. Y. (k/fed) } & \multicolumn{2}{|c|}{ B. W. (gm) } \\
\hline Genotypes & Old & New & Old & New & Old & New \\
\hline G.85 & 10.46 & 8.94 & 13.25 & 10.95 & 2.84 & 2.85 \\
\hline G.86 & 9.83 & 9.39 & 12.48 & 11.76 & 2.95 & 2.88 \\
\hline G.89 & 9.64 & 8.90 & 11.41 & 10.26 & 2.76 & 2.84 \\
\hline G.89 x G.86 & 9.76 & 7.81 & 12.06 & 9.32 & 2.78 & 2.84 \\
\hline G.89 x Pima S-6 & 9.98 & 7.90 & 12.64 & 9.71 & 2.61 & 2.74 \\
\hline L.S.D. (I) & ---- & 1.01 & 1.15 & 1.05 & 0.13 & ---- \\
\hline L.S.D. (M) & ---- & 1.23 & ---- & 1.49 & 0.12 & ---- \\
\hline L.S.D. (C) & \multicolumn{2}{|c|}{---} & \multicolumn{2}{|c|}{1.11} & \multicolumn{2}{|c|}{---- } \\
\hline Traits & \multicolumn{2}{|c|}{ L.P. (\%) } & \multicolumn{2}{|c|}{ S. I. (gm) } & & \\
\hline G.85 & 40.11 & 38.81 & 9.72 & 9.65 & & \\
\hline G.86 & 40.10 & 39.73 & 10.26 & 10.28 & & \\
\hline G.89 & 37.68 & 36.63 & 9.91 & 9.88 & & \\
\hline \multirow{5}{*}{$\begin{array}{l}\text { G.89 x G.86 } \\
\text { G.89 x Pima S-6 } \\
\text { L.S.D. (I) } \\
\text { L.S.D. (M) } \\
\text { L.S.D. (C) }\end{array}$} & 39.23 & 37.82 & 9.82 & 10.18 & & \\
\hline & 40.29 & 38.28 & 9.08 & 10.24 & & \\
\hline & 0.90 & 0.96 & 0.50 & ---- & & \\
\hline & 1.03 & 1.03 & \multirow{2}{*}{\multicolumn{2}{|c|}{0.66}} & & \\
\hline & & & & & & \\
\hline
\end{tabular}

\subsubsection{Modified analysis (two seasons)}

The analysis of variance revealed significant variation due to partitioning of the genotypes, (Table 6). Genotypes within both the old and new locations exhibited significant differences for yield components except (S.I.) within the new location. Significant variation due to the old vs. the new was observed for (S.C.Y.) and (L.C.Y.) indicating that yield was more affected by different locations.

\subsubsection{Combined analysis (two seasons)}

The analysis of variance showed significant variation due to locations and (genotypes $\mathrm{x}$ locations), (Table 6). Significant difference on locations was observed for yield indicating that yield was highly affected by locations than yield 
Table (5): Mean squares of yield and its components for individual location (two seasons).

\begin{tabular}{|c|c|c|c|c|c|c|}
\hline \multicolumn{7}{|c|}{ Old location (Middle Delta) } \\
\hline \multicolumn{2}{|l|}{ Traits } & \multirow{2}{*}{$\begin{array}{l}\text { S.C.Y. } \\
\text { (k/fed) }\end{array}$} & \multirow{2}{*}{$\begin{array}{l}\text { L.C.Y. } \\
\text { (k/fed) }\end{array}$} & \multirow{2}{*}{$\begin{array}{l}\text { B.W. } \\
\text { (gm) }\end{array}$} & \multirow{2}{*}{$\begin{array}{c}\text { L.P. } \\
(\%)\end{array}$} & \multirow{2}{*}{$\begin{array}{l}\text { S. I. } \\
\text { (gm) }\end{array}$} \\
\hline Source of variation & d.f. & & & & & \\
\hline Replications & 15 & $15.11 * *$ & $17.79 * *$ & $0.179 * *$ & $3.31 * *$ & $4.82 * *$ \\
\hline Genotypes & 4 & 3.77 & 4.23 & $0.232 * *$ & $14.82 * *$ & $1.75^{* *}$ \\
\hline Cultivars (C) & 2 & 0.481 & 2.03 & $0.146 * *$ & $27.34 * *$ & $1.09 *$ \\
\hline Hybrids (H) & 1 & $12.95 *$ & $12.63^{*}$ & $0.430 * *$ & $3.91 *$ & $4.44 * *$ \\
\hline C. vs. H. & 1 & 1.17 & 0.241 & $0.205 * *$ & 0.658 & 0.388 \\
\hline Experimental error & 60 & 1.85 & 2.30 & 0.021 & 0.873 & 0.277 \\
\hline \multicolumn{7}{|c|}{ New location (North Delta) } \\
\hline \multicolumn{2}{|l|}{ Traits } & S.C.Y. & L.C.Y. & B.W. & L. $P$. & S. I. \\
\hline Source of variation & d.f. & $(\mathbf{k} / \mathbf{f e d})$ & $(\mathrm{k} / \mathrm{fed})$ & $(\mathrm{gm})$ & $(\%)$ & (gm) \\
\hline Replications & 15 & $13.97 * *$ & $25.06 * *$ & $0.270 * *$ & $4.72 * *$ & $2.09 * *$ \\
\hline Genotypes & 4 & 1.46 & 5.13 & $0.116^{* *}$ & $6.76^{* *}$ & $1.18^{* *}$ \\
\hline Cultivars (C) & 2 & 1.59 & 6.79 & $0.110 * *$ & $13.13 * *$ & $2.10 * *$ \\
\hline Hybrids (H) & 1 & 1.74 & 4.46 & 0.014 & 0.383 & 0.500 \\
\hline C. vs. H. & 1 & 0.899 & 2.48 & $0.231 * *$ & 0.401 & 0.027 \\
\hline Experimental error & 60 & 1.88 & 2.55 & 0.021 & 1.14 & 0.322 \\
\hline
\end{tabular}

$*$,** Significant at 0.05 and 0.01 levels, respectively.

Table (6): Mean squares of yield and its components for two ways of analysis randomized complete block design (two seasons).

\begin{tabular}{|c|c|c|c|c|c|c|}
\hline \multicolumn{7}{|c|}{ Modified analysis } \\
\hline \multicolumn{2}{|l|}{ Traits } & \multirow{2}{*}{$\begin{array}{l}\text { S.C.Y. } \\
\text { (k/fed) }\end{array}$} & \multirow{2}{*}{$\begin{array}{l}\text { L.C.Y. } \\
\text { (k/fed) }\end{array}$} & \multirow{2}{*}{$\begin{array}{l}\text { B.W. } \\
\text { (gm) }\end{array}$} & \multirow{2}{*}{$\begin{array}{l}\text { L. P. } \\
(\%)\end{array}$} & \multirow{2}{*}{$\begin{array}{l}\text { S. I. } \\
\text { (gm) }\end{array}$} \\
\hline Source of variation & d.f. & & & & & \\
\hline Replications & 15 & $25.24 * *$ & $35.62 * *$ & $0.328 * *$ & 0.399 & $4.92 * *$ \\
\hline Genotypes & 9 & $16.80 * *$ & $23.88 * *$ & $0.155^{* *}$ & $10.05 * *$ & $1.47 * *$ \\
\hline Within old location & 4 & 3.77 & 4.23 & $0.231 * *$ & $14.81 * *$ & $1.75^{* *}$ \\
\hline Within new location & 4 & 1.46 & 5.13 & $0.116 * *$ & $6.76 * *$ & 1.18 \\
\hline Old vs. New & 1 & $130.25^{* *}$ & $177.45^{* *}$ & 0.004 & 4.16 & 1.49 \\
\hline Experimental error & 135 & 2.08 & 2.95 & 0.032 & 1.74 & 0.487 \\
\hline \multicolumn{7}{|c|}{ Combined analysis } \\
\hline \multicolumn{2}{|l|}{ Traits } & \multirow{2}{*}{$\begin{array}{l}\text { S.C.Y. } \\
\text { (k/fed) }\end{array}$} & \multirow{2}{*}{$\begin{array}{l}\text { L.C.Y. } \\
\text { (k/fed) }\end{array}$} & \multirow{2}{*}{$\begin{array}{l}\text { B.W. } \\
\text { (gm) }\end{array}$} & \multirow{2}{*}{$\begin{array}{l}\text { L. P. } \\
(\%)\end{array}$} & \multirow{2}{*}{$\begin{array}{l}\text { S. I. } \\
\text { (gm) }\end{array}$} \\
\hline Source of variation & d.f. & & & & & \\
\hline Locations (L) & 1 & $130.25^{* *}$ & $177.45^{* *}$ & 0.004 & 4.16 & 1.49 \\
\hline Rep. / Locations & 30 & 14.54 & 21.42 & 0.224 & 4.02 & 3.45 \\
\hline Genotypes (G) & 4 & 3.44 & $7.73 *$ & $0.311 * *$ & $20.44 * *$ & $1.79 * *$ \\
\hline $\mathbf{G} \times \mathbf{L}$ & 4 & 1.78 & 1.63 & 0.037 & 1.48 & $1.15^{* *}$ \\
\hline Experimental error & 120 & 1.87 & 2.42 & 0.021 & 1.01 & 0.300 \\
\hline
\end{tabular}

$*$,** Significant at 0.05 and 0.01 levels, respectively.

Table (7): Means of yield and its components (two seasons).

\begin{tabular}{|c|c|c|c|c|c|c|}
\hline Traits & \multicolumn{2}{|c|}{ S. C. Y. (k/fed) } & \multicolumn{2}{|c|}{ L. C. Y. (k/fed) } & \multicolumn{2}{|c|}{ B. W. (gm) } \\
\hline Genotypes & Old & New & Old & New & Old & New \\
\hline G.85 & 11.38 & 9.53 & 14.12 & 11.75 & 2.98 & 3.00 \\
\hline G.86 & 11.32 & 10.16 & 14.30 & 12.84 & 3.09 & 3.09 \\
\hline G.89 & 11.65 & 9.80 & 13.61 & 11.69 & 2.90 & 2.92 \\
\hline G.89 x G.86 & 11.06 & 9.38 & 13.50 & 11.36 & 3.00 & 2.91 \\
\hline G.89 x Pima S-6 & 12.33 & 9.85 & 14.75 & 12.11 & 2.77 & 2.87 \\
\hline L.S.D. (I) & 0.96 & $\begin{array}{l}--- \\
-\end{array}$ & 1.07 & ---- & 0.10 & 0.10 \\
\hline L.S.D. (M) & ---- & ---- & ---- & ---- & 0.12 & 0.12 \\
\hline L.S.D. (C) & \multicolumn{2}{|c|}{----} & \multicolumn{2}{|c|}{---- } & \multicolumn{2}{|c|}{---- } \\
\hline Traits & \multicolumn{2}{|c|}{ L. P. (\%) } & \multicolumn{2}{|c|}{ S. I. (gm) } & & \\
\hline G.85 & 39.36 & 39.02 & 10.71 & 10.32 & & \\
\hline G.86 & 40.01 & 39.51 & 10.95 & 10.84 & & \\
\hline G.89 & 37.49 & 37.75 & 10.43 & 10.14 & & \\
\hline G.89 x G.86 & 38.79 & 38.51 & 10.93 & 10.35 & & \\
\hline \multirow{4}{*}{$\begin{array}{l}\text { G.89 x Pima S-6 } \\
\text { L.S.D. (I) } \\
\text { L.S.D. (M) } \\
\text { L.S.D. (C) }\end{array}$} & 39.49 & 38.73 & 10.18 & 10.60 & & \\
\hline & 0.66 & 0.75 & 0.37 & 0.40 & & \\
\hline & 0.92 & 0.92 & 0.49 & --- & & \\
\hline & & & \multicolumn{2}{|c|}{0.38} & & \\
\hline
\end{tabular}

---- : Not significant at $5 \%$. (I) = Individual location. $(\mathrm{M})=$ Modified analysis. $(\mathrm{C})=$ Combined analysis. 
components. Significant variation due to (genotypes $\mathrm{x}$ locations) was detected for only one trait, (S.I.).

\subsection{Fiber properties}

\subsubsection{Analysis of individual location (one season)}

Each location considered 2 replications and analysis of normal randomized complete block design. The analysis of variance revealed significant variation due to replications, genotypes and partitioning of genotypes, (Table 8). In both locations, significant difference due to replications (variance within each location) was observed for (F.L.) in the first season indicating that (F.L.) was more affected by different environments within each location than other traits. In the first season, significant variation due to the genotypes and partitioning of them were detected on (F.L) and (Mic.) in both locations except cultivars and cultivars vs hybrids with respect to (F.L.) in the old location. In contrast, in the second season, genotypes and partitioning of them exhibited non significant differences with respect to all traits in all locations except genotypes and hybrids for (St.) and cultivars for (F.L.) in the old location. The results indicated that responses of genotypes and partitioning of them were similar in both locations.

\subsubsection{Modified analysis (one season)}

The analysis of variance showed significant variation due to partitioning of genotypes, (Table 9). Significant variation due to genotypes within old location was detected on (Mic.) in the first season and (F.L.) in the second season. Genotypes within new location exhibited significant variation for two traits, viz. (F.L.) and (Mic.) in the first season. Significant difference due to the old vs. the new location was observed only for one trait, i.e. (Mic.) in 2004 season declaring that both (F.L.) and (St.) were not affected by different location.

Table (8): Mean Squares of fiber properties for individual location (one season),

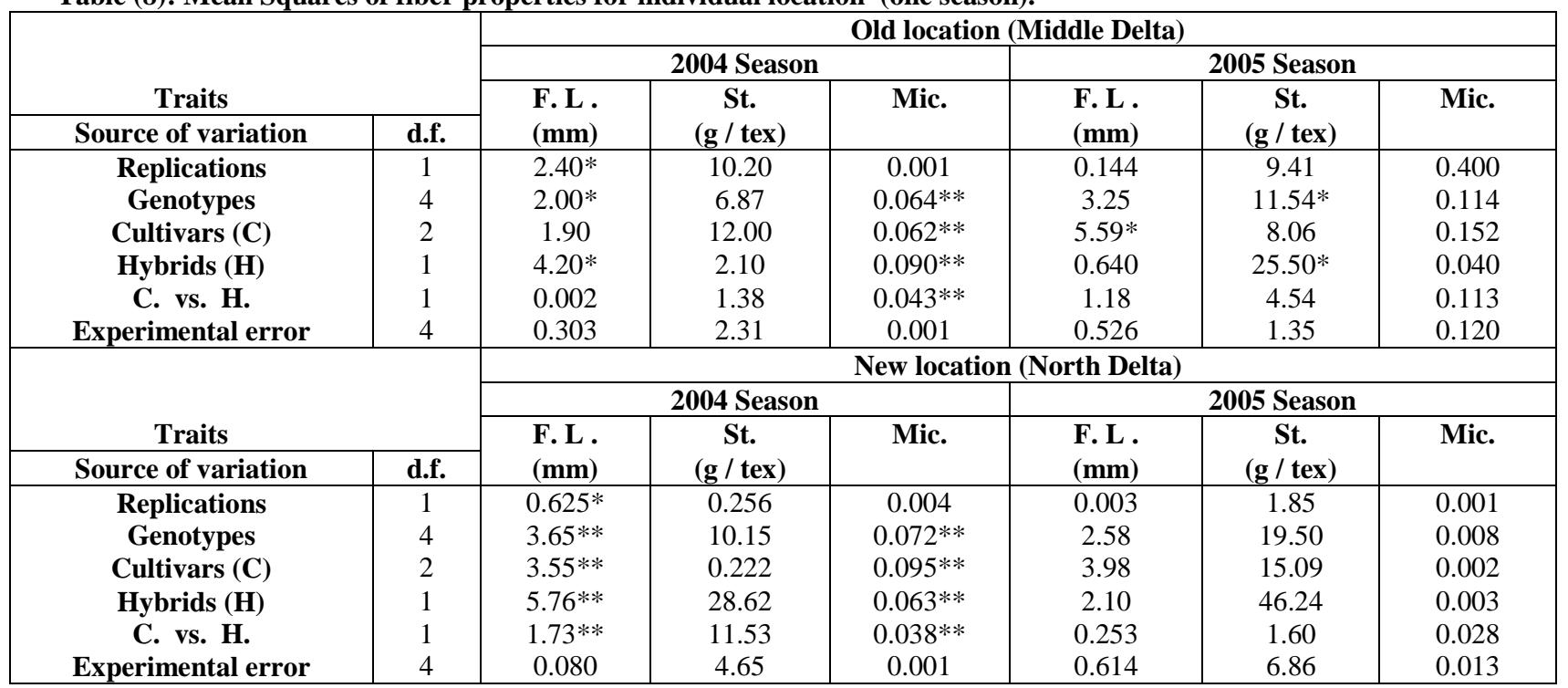

*, ** Significant at 0.05 and 0.01 levels, respectively.

\subsubsection{Combined analysis (one season)}

The analysis of variance revealed significant differences due to locations and (genotypes $\mathrm{x}$ locations), (Table 9). Significant variation due to locations was observed for (Mic.) in 2004 and (F.L.) in 2005 season. The present results indicated that (St.) was not affected by locations. Significant variation due to genotypes $\mathrm{x}$ locations was detected for only one trait, (Mic) in the first season. The results indicated that both (F.L.) and (St.) were not affected by the interaction (genotypes $\mathrm{x}$ locations).

\subsubsection{Analysis of individual location (two seasons)}

Each location considered 4 replications and analysis for normal randomized complete block design. The analysis of variance revealed significant variation due to replications, genotypes and partitioning of genotypes, (Table 11). Significant variation due to replications was observed for (St.) in the old location and (Mic.) in both locations. The results indicated significance due to replications according to different environments within each location. Significant variations due to cultivars and hybrids were 
Table (9): Mean squares of fiber properties for two ways of analysis of randomized complete block design (one season).

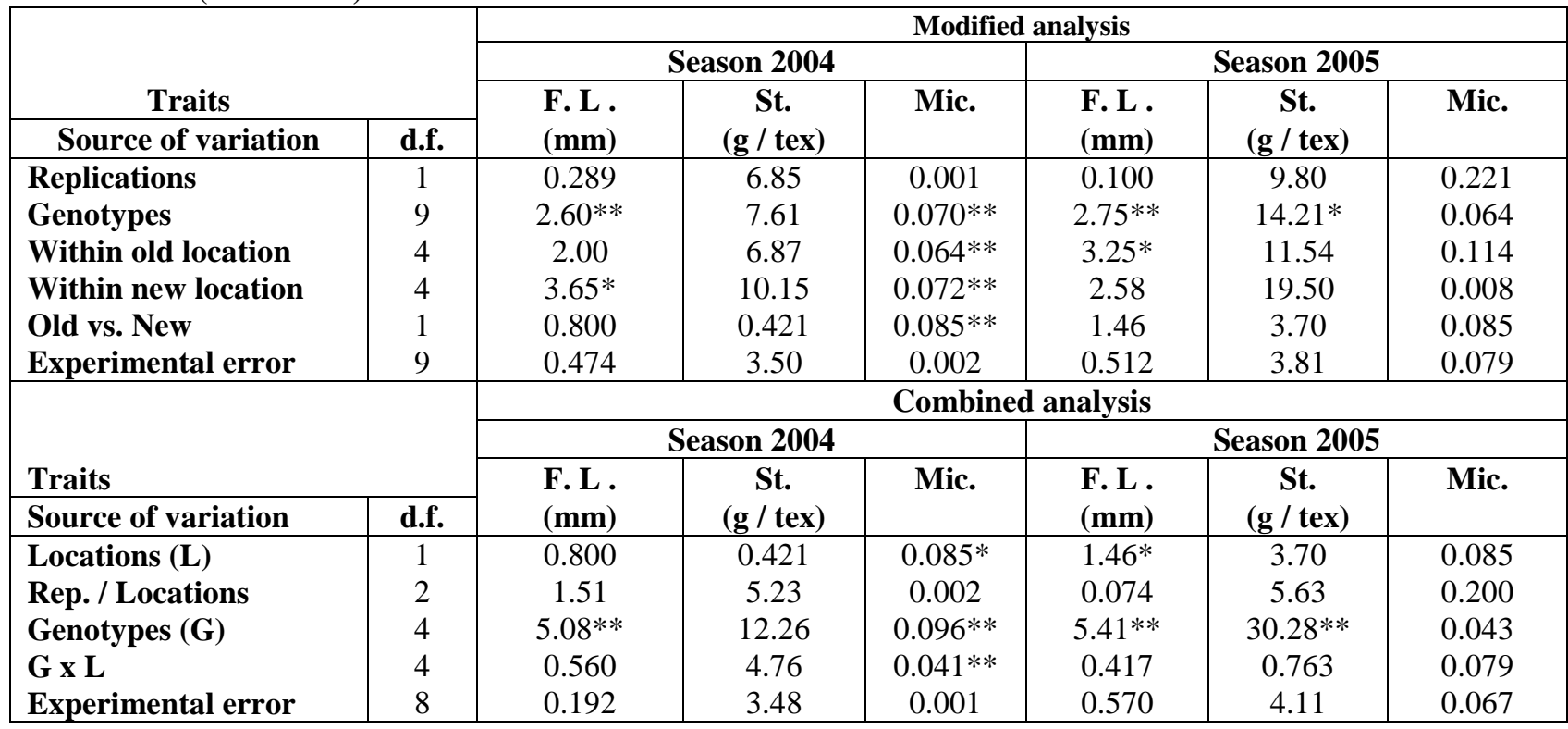

$*$,** Significant at 0.05 and 0.01 levels, respectively.

Table (10) Means of fiber properties (one season).

\begin{tabular}{|c|c|c|c|c|c|c|}
\hline \multicolumn{7}{|c|}{ Season 2004} \\
\hline \multirow[b]{2}{*}{ Genotypes } & \multicolumn{2}{|c|}{ F. L. (mm) } & \multicolumn{2}{|c|}{ St. (g/tex) } & \multicolumn{2}{|c|}{ Mic. } \\
\hline & Old & New & Old & New & Old & New \\
\hline G.85 & 31.25 & 30.30 & 40.10 & 40.30 & 4.40 & 4.20 \\
\hline G.86 & 32.70 & 32.92 & 43.35 & 40.75 & 4.75 & 4.60 \\
\hline G.89 & 30.85 & 31.40 & 38.55 & 40.10 & 4.60 & 4.55 \\
\hline G.89 x G.86 & 32.65 & 31.90 & 42.15 & 45.25 & 4.60 & 4.20 \\
\hline G.89 x Pima S-6 & 30.60 & 29.50 & 40.70 & 39.90 & 4.30 & 4.45 \\
\hline L.S.D. (I) & 1.53 & 0.78 & ---- & ---- & 0.09 & 0.11 \\
\hline L.S.D. (M) & ---- & 1.56 & ---- & ---- & 0.09 & 0.09 \\
\hline L.S.D. (C) & \multicolumn{2}{|c|}{--- } & & & \multicolumn{2}{|c|}{0.07} \\
\hline \multicolumn{7}{|c|}{2005 Season } \\
\hline \multirow[b]{2}{*}{ Genotypes } & \multicolumn{2}{|c|}{ F. L. (mm) } & \multicolumn{2}{|c|}{ St. (g/tex) } & \multicolumn{2}{|c|}{ Mic. } \\
\hline & Old & New & Old & New & Old & New \\
\hline G.85 & 29.65 & 29.05 & 38.70 & 39.25 & 4.35 & 4.05 \\
\hline G.86 & 32.90 & 31.85 & 41.90 & 43.85 & 4.05 & 4.05 \\
\hline G.89 & 31.95 & 30.75 & 38.20 & 38.95 & 3.80 & 4.10 \\
\hline G.89 x G.86 & 31.20 & 31.60 & 43.50 & 44.90 & 3.95 & 4.20 \\
\hline G.89 x Pima S-6 & 30.40 & 30.15 & 38.45 & 38.10 & 3.75 & 4.15 \\
\hline L.S.D. (I) & 2.02 & ---- & 3.23 & ---- & $\begin{array}{l}--- \\
-\end{array}$ & ---- \\
\hline L.S.D. (M) & 1.62 & ---- & ---- & ---- & ---- & ---- \\
\hline L.S.D. (C) & \multicolumn{2}{|c|}{--- } & \multicolumn{2}{|c|}{---} & \multicolumn{2}{|c|}{---- } \\
\hline
\end{tabular}

---- : Not significant at $5 \%$. $\quad A=$ Individual location. $(M)=$ Modified analysis. $(C)=$ Combined analysis.

Table (11): Mean squares of fiber properties for individual location (two seasons).

\begin{tabular}{|c|c|c|c|c|c|c|c|}
\hline \multirow{2}{*}{\multicolumn{2}{|c|}{ Traits }} & \multicolumn{3}{|c|}{ Old location (Middle Delta) } & \multicolumn{3}{|c|}{ New location (North Delta) } \\
\hline & & \multirow{2}{*}{$\begin{array}{l}\text { F. L. } \\
(\mathbf{m m})\end{array}$} & \multirow{2}{*}{$\begin{array}{c}\text { St. } \\
(\mathrm{g} / \mathrm{tex})\end{array}$} & \multirow[t]{2}{*}{ Mic. } & \multirow{2}{*}{$\begin{array}{l}\text { F. L. } \\
(\mathbf{m m})\end{array}$} & \multirow{2}{*}{$\begin{array}{c}\text { St. } \\
\text { (g/tex) }\end{array}$} & \multirow[t]{2}{*}{ Mic. } \\
\hline Source of variation & d.f. & & & & & & \\
\hline Replications & 3 & 1.10 & $7.66^{*}$ & $0.638 * *$ & 0.678 & 0.806 & $0.142 * *$ \\
\hline Genotypes & 4 & $3.95 * *$ & $16.48 * *$ & 0.092 & $5.65^{* *}$ & $25.88 *$ & 0.032 \\
\hline Cultivars (C) & 2 & $5.59 * *$ & $19.68 * *$ & 0.048 & $7.43 * *$ & 9.43 & 0.053 \\
\hline Hybrids (H) & 1 & $4.06 *$ & $21.13 * *$ & 0.125 & $7.41 * *$ & $73.81 * *$ & 0.020 \\
\hline C. vs. H. & 1 & 0.545 & 5.46 & 0.147 & 0.331 & 10.86 & 0.001 \\
\hline Experimental error & 12 & 0.710 & 1.87 & 0.069 & 0.423 & 5.09 & 0.021 \\
\hline
\end{tabular}

*,** Significant at 0.05 and 0.01 levels, respectively.

detected on (F.L) and (St.) in both locations except cultivars with respect to (St.) in the new location.

\subsubsection{Modified analysis (two seasons)}

The analysis of variance revealed significant variation due to partitioning of the genotypes, 
(Table 12). The genotypes within both old and new locations exhibited significant differences for all traits except (Mic.). Old vs. new exhibited nonsignificant differences for all traits indicating that fiber properties were not affected by different locations.

\subsubsection{Combined analysis (two seasons)}

Both locations and (genotypes $\mathrm{x}$ locations) exhibiting non-significant differences for all traits. This indicated that fiber properties were not affected by different locations and (genotypes $\mathrm{x}$ locations), (Table 12).

\subsection{Comparison between modified and combined analyses}

Modified surpassed combined because it does not need calculating homogeneity test of variances (Bartlett test) before the start of analysis. Modified depends on increasing the number of genotypes, while combined depends on increasing the number of replications. Modified depends on partition of genotypes to estimate both locations and genotypes x locations variances, while combined used two various steps to calculate the same variances. Degree of freedom of experimental error in modified was bigger than combined. Although two ways of analyses calculate the same value of locations variance but they exhibited different results of significant variation due to different values of $\mathrm{F}$ Table of them, which depends on degrees of freedom of error. Results exhibited that sum two groups of genotypes variances (within old location + within new location) in modified analysis equal sum of both genotypes and genotypes $\mathrm{x}$ locations variances in combined analysis.

Table (12): Mean squares of fiber properties for two ways of analysis of randomized complete block design (two seasons).

\begin{tabular}{|c|c|c|c|c|}
\hline \multirow{2}{*}{\multicolumn{2}{|c|}{ Traits }} & \multicolumn{3}{|c|}{ Modified analysis } \\
\hline & & \multirow{2}{*}{$\begin{array}{l}\text { F. L. } \\
(\mathbf{m m})\end{array}$} & \multirow{2}{*}{$\begin{array}{c}\text { St. } \\
\text { (g/tex) }\end{array}$} & \multirow[t]{2}{*}{ Mic. } \\
\hline Source of variation & d.f. & & & \\
\hline Replications & 3 & 0.833 & 6.50 & $0.662 * *$ \\
\hline Genotypes & 9 & $4.51 * *$ & $19.20 * *$ & 0.055 \\
\hline Within old location & 4 & $3.95 * *$ & $16.48 * *$ & 0.092 \\
\hline Within new location & 4 & $5.65 * *$ & $25.88 * *$ & 0.032 \\
\hline Old vs. New & 1 & 2.21 & 3.31 & 0.001 \\
\hline Experimental error & 27 & 0.608 & 3.31 & 0.053 \\
\hline \multirow{2}{*}{\multicolumn{2}{|c|}{ Traits }} & \multicolumn{3}{|c|}{ Combined analysis } \\
\hline & & \multirow{2}{*}{$\begin{array}{l}\text { F. L } \\
(\mathbf{m m})\end{array}$} & \multirow{2}{*}{$\begin{array}{c}\text { St. } \\
\text { (g/tex) }\end{array}$} & \multirow[t]{2}{*}{ Mic. } \\
\hline $\begin{array}{c}\text { Source of variation } \\
\end{array}$ & d.f & & & \\
\hline Locations $(\mathbf{L})$ & 1 & 2.21 & 3.31 & 0.001 \\
\hline Rep. / Locations & 6 & 0.890 & 4.23 & 0.390 \\
\hline Genotypes (G) & 4 & $9.47 * *$ & $39.71 * *$ & 0.041 \\
\hline $\mathbf{G} \times \mathbf{L}$ & 4 & 0.124 & 2.65 & 0.083 \\
\hline Experimental error & 24 & 0.566 & 3.48 & 0.045 \\
\hline
\end{tabular}

*, ** Significant at 0.05 and 0.01 levels, respectively.

Table (13) Means of fiber properties (two seasons).

\begin{tabular}{|c|c|c|c|c|c|c|}
\hline \multirow{2}{*}{ Genotypes } & \multicolumn{2}{|c|}{ F. L. (mm) } & \multicolumn{2}{c|}{ St. (g/tex) } & \multicolumn{2}{c|}{ Mic. } \\
\cline { 2 - 7 } G.85 & Old & New & Old & New & Old & New \\
G.86 & 30.45 & 29.68 & 39.40 & 39.78 & 4.38 & 4.13 \\
G.89 & 32.80 & 32.40 & 42.63 & 42.30 & 4.40 & 4.32 \\
G.89 x G.86 & 31.40 & 31.07 & 38.37 & 39.53 & 4.20 & 4.32 \\
G.89 x Pima S-6 & 31.92 & 31.75 & 42.83 & 45.08 & 4.28 & 4.20 \\
L.S.D. (I) & 30.50 & 29.82 & 39.58 & 39.00 & 4.03 & 4.30 \\
\cline { 2 - 7 } L.S.D. (M) & 1.30 & 1.00 & 2.11 & 3.48 & --- & ---- \\
L.S.D. (C) & 1.13 & 1.13 & 2.64 & 2.64 & --- & -- \\
\hline
\end{tabular}

---- : Not significant at $5 \% . \quad(\mathrm{I})=$ Individual location. $(\mathrm{M})=$ Modified analysis. $(\mathrm{C})=$ Combined analysis.

\section{REFERENCES}

Bailey N.T. (1994).Statistical Methods in Biology.Third Edit. Cambride University Press, Cambridge, England.

Cochran W.G. and Cox G. M. (1950). Experimental Designs. John Wiley and Sons, New York., U.S.A.
El Oraby S.H. (2003). Comparative study between some newly released Egyptian cotton crosses and some commercial cultivars grown in different locations. Ph.D.Thesis, Kafr El Sheikh, Tanta Univ., Egypt. 
Federer W. T. (1955). Experimental Design Theory and Application. The Macmilan Company New York, U.S.A.

Gomez K. A. and Gomez A. A. (1984). Statistical Procedures for Agricultural Research. Wiley, New York, U.S.A..

Idris H. A. (2002). Models for evaluating Egyptian cotton responses to environmental variables. Ph. D. Thesis, Fac., of Agric., Cairo Univ., Giza, Egypt.

Idris H. A. (2005). Evaluating some Egyptian cotton genotypes by using two steps of randomized complete block design and cluster analysis. Bull. Fac. Agric., Cairo Univ., 56: 767-778.

Mohamed S. A. (2005). Evaluation of some genotypes in Egyptian cotton at different locations. Msc. Thesis, Kafr El Sheikh, Tanta Univ., Egypt.

Roger G. P. (1994). Agricultural Field Experiments Design and Analysis. Marcel Dekker, Inc. New york, U.S.A.

Steel R. G. and Torrie J. H. (1980). Principles and Procedures of Statistics. Second Edit., McGraw Hill Book Co. New York, U.S.A.

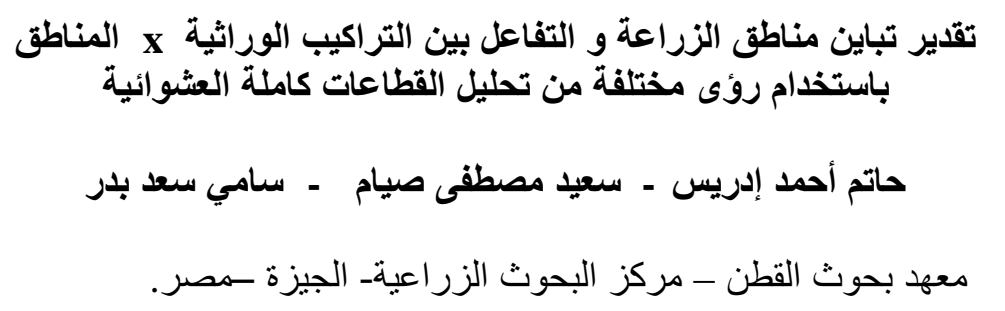

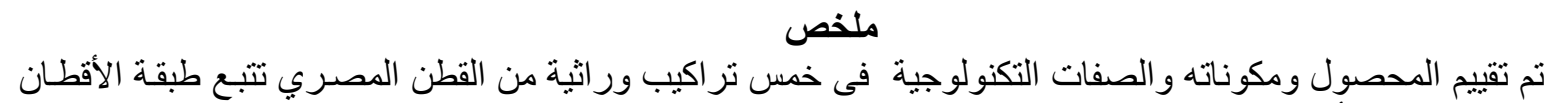

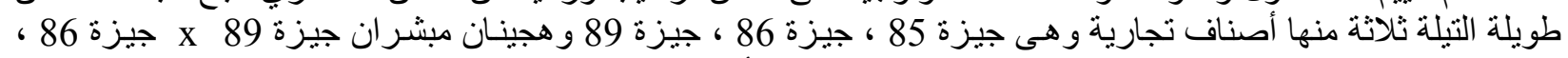

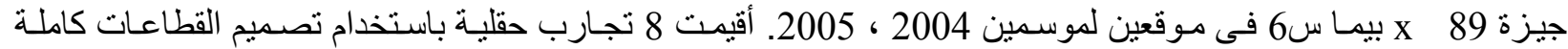
العشو ائية في 4 مكرر ات لكل تجربة.

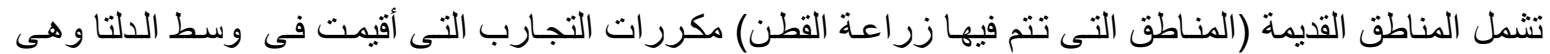

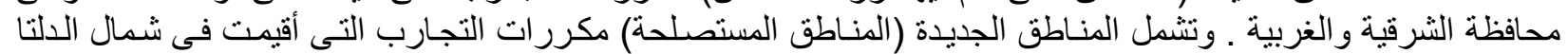

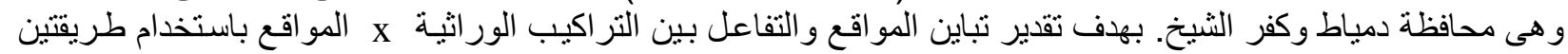
من تحليل القطاعات كاملة العشو ائية.

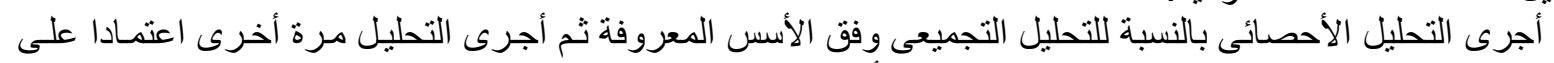

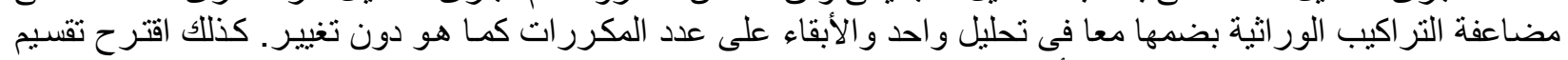

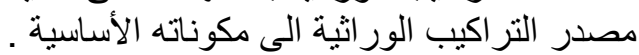

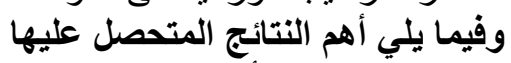

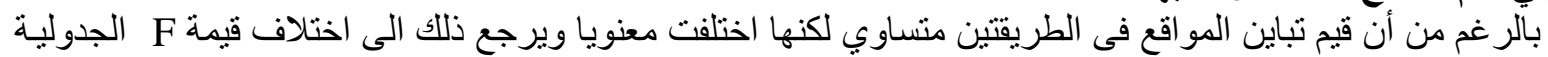

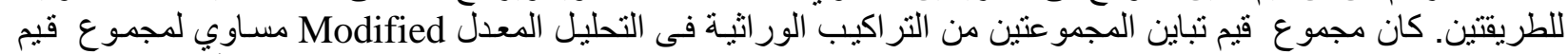

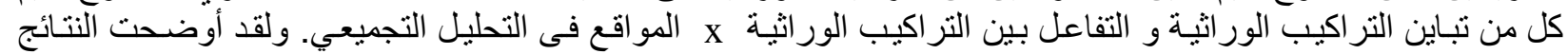

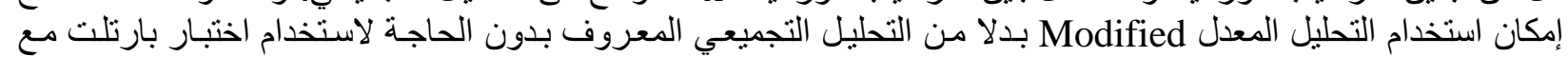

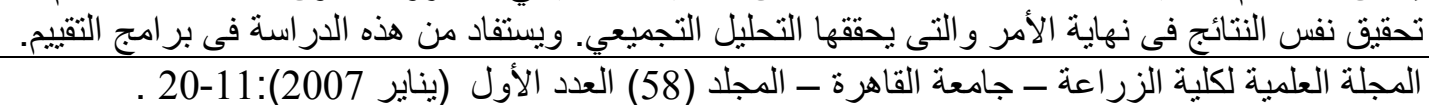

